\title{
Les pratiques de formation dans l'intérim
}

Formes, évolutions et limites

Training practices in the temporary work sector : forces, evolutions and limits

\section{Rachid Belkacem et Hervé Lhotel}

\section{(2) OpenEdition}

\section{Journals}

Édition électronique

URL : http://journals.openedition.org/travailemploi/5844

DOI : 10.4000/travailemploi.5844

ISSN : 1775-416X

Éditeur

DARES - Ministère du Travail

Édition imprimée

Date de publication : 15 décembre 2012

Pagination : 49-63

ISSN : 0224-4365

Référence électronique

Rachid Belkacem et Hervé Lhotel, « Les pratiques de formation dans l'intérim », Travail et Emploi [En ligne], 132 | octobre-décembre 2012, mis en ligne le 01 décembre 2014, consulté le 19 avril 2019. URL : http://journals.openedition.org/travailemploi/5844; DOI : 10.4000/travailemploi.5844 


\title{
Les pratiques de formation dans l'intérim : formes, évolutions et limites
}

\author{
Rachid Belkacem ${ }^{(*)}$, Hervé Lhotel(**)
}

\begin{abstract}
Cet article s'intéresse aux politiques de formation dans l'intérim en France. En 1983 cette branche s'est dotée d'un Fonds d'assurance formation, et depuis lors, le cadre institutionnel de la formation a été négocié entre les partenaires sociaux. L'intérim s'est approprié des dispositifs de formation de droit commun (en particulier la formation en alternance) en les adaptant puis a élaboré les siens. Ce cadre a permis l'impulsion à la fois de la croissance du nombre d'actions de formation et de celle de la dépense financière. La dégradation de la conjoncture économique à partir de 2008 a eu des effets extrêmement négatifs sur les pratiques de formation. D’après les données analysées, les formations sont en tendance de courte, voire de très courte durée. Les formations longues sont réservées aux intérimaires les plus expérimentés et quasi-permanents. Pour partie, ceci s'explique par le mode de fonctionnement de la branche et les formes d'usage de la main-d'œuvre intérimaire.
\end{abstract}

Le travail intérimaire est devenu aujourd'hui un instrument de gestion de la main-d'œuvre très prisé par les entreprises des secteurs de l'industrie, du bâtiment et des travaux publics. Le recours aux agences d'intérim leur permet d'apporter des solutions à des problèmes tels qu'une absence de personnel, un accroissement d'activité, la sélection de travailleurs en amont d'un recrutement, voire des actions de formation. Selon une étude de la Direction de l'animation de la recherche, des études et des statistiques (Dares) menée par Jean FinOT (2012), 576100 personnes en équivalent temps plein occupent régulièrement un emploi intérimaire en 2011. Chaque année, environ deux millions de personnes réalisent au moins une mission d'intérim et transitent ainsi par les services des agences et entreprises de travail temporaire (ETT). Le développement de cette activité a soulevé et soulève toujours beaucoup de questions. C'est celle de la formation des travailleurs intérimaires qui nous intéresse ici. Elle recèle une acuité toute particulière en raison de la situation de précarité dans laquelle se trouvent la plupart d'entre eux, conséquence de la tendance à la discontinuité des relations salariales et à l'irrégularité des revenus salariaux qui en découle. En effet, l'intérimaire n'est salarié de l'ETT que pendant sa mission d'intérim. D'après FINOT (2011), la durée moyenne des détachements s'établit à seulement 1,7 semaine en 2010; un intérimaire sur deux a été en mission moins de deux mois (1,5 précisément) cette année-là. La relation salariale qui unit l'intérimaire à son employeur (l'ETT) est ainsi généralement de

(*) Université de Lorraine, Laboratoire lorrain des sciences sociales; rachid.belkacem@iut-longwy.uhp-nancy.fr

(**) Université de Lorraine, Laboratoire lorrain des sciences sociales, Cereq; lhotel@univ-nancy2.fr courte durée, sauf dans le cas minoritaire d'enchaînement continu de missions. Comment peut alors s'organiser la formation dans cette branche d'activité? Et quels intérimaires sont formés? Selon le rapport 2011 de la profession (PRISME $\left.{ }^{(1)}, 2011\right)$, un peu plus des trois-quarts des intérimaires $(77 \%)$ sont ouvriers, et la moitié d'entre eux sont reconnus peu ou pas qualifiés au regard des missions proposées. La formation peut donc représenter un enjeu important pour ces travailleurs qui, pour la plupart, sont en recherche d'insertion ou de réinsertion professionnelle. Au niveau national, d'après les données issues de l'exploitation des déclarations fiscales 24-83(2), traitées par le Cereq ${ }^{(3)}$, un quart des intérimaires a participé en 2002 à une action de formation professionnelle continue s'inscrivant dans le plan de formation de l'entreprise (Möвus, 2006, p. 26). Qu'en est-il aujourd'hui? De quels types de formation s'agit-il? Quels sont les dispositifs mis en œuvre?

En France, c'est en 1983 que vont se dessiner les fondements du cadre institutionnel de la formation dans l'intérim avec la création d'un Fonds d'assurance formation pour le travail temporaire (FAF-TT). Il s'en est suivi une augmentation des actions de formation à destination de ces travailleurs et une hausse spectaculaire du montant

(1) Professionnels de l'intérim, services et des métiers de l'emploi qui est l'organisation patronale de la profession.

(2) Il s'agit du formulaire «24-83» de la déclaration fiscale annuelle sur le financement de la formation professionnelle continue par les entreprises de dix salariés et plus.

(3) Centre d'études et de recherches sur les qualifications. 


\section{Encadré 1}

\section{Trois principales sources d'information}

1. Des données internes au secteur de l'intérim, produites notamment par le Fonds d'assurance formation du travail temporaire (FAF-TT), par l'Observatoire des métiers et des emplois (OME) ainsi que les données publiées par la profession dans les rapports économiques du secteur (cf. SETT [Syndicat des entreprises du travail temporaire qui a précédé le PRISME] et PRISME, rapports économique et social). Les informations présentées dans cet article pour analyser les caractéristiques sociodémographiques des bénéficiaires des mesures de formation en 2010 sont issues des publications du FAF-TT. Elles concernent l'ensemble des bénéficiaires des mesures financées cette année-là par l'organisme et portent sur le genre, l'âge, le niveau initial de formation, le type de validation et la durée moyenne des formations. Les informations sur les effets de ces dispositifs sur les trajectoires professionnelles émanent d'enquêtes spécifiques commanditées par le FAF-TT (2011a) : les envois de questionnaires auprès d'un échantillon de bénéficiaires de ces contrats se sont achevés entre le 22 juin 2009 et le 3 juillet 2010. Ils ont couvert précisément 2669 bénéficiaires d'un contrat de professionnalisation (498 ont répondu, ce qui représente un taux de réponse après relance de $19 \%$ ); 1104 bénéficiaires d'un contrat de développement professionnel intérimaire (taux de réponse : $24 \%$ ); 2851 bénéficiaires du congé individuel de formation (1172 intérimaires ont répondu; taux de réponse de $41 \%)$.

2. Des données issues de monographies de grandes ETT réalisées quasiment en continu depuis 1997 : Manpower, Védior-Bis, Adecco, Plus Intérim (BeLKACEM, 2002; BeLKACEM, MıchoN, 2011). Ces monographies étaient, schématiquement, organisées autour des points suivants : identification (présentation générale de l'entreprise), données historiques (grandes étapes depuis la création de l'entreprise), activité stratégique (dont les politiques commerciales), politique de gestion des travailleurs permanents, politique de gestion des intérimaires (salaires, formation, détachements, fidélisation, placements, etc.).

3. Par ailleurs, deux vagues d'enquêtes auprès de responsables d'agences d'intérim ont été réalisées à l'occasion de travaux de recherche sur l'intérim et ont traité la question de la formation des intérimaires :

- En 2001, douze entretiens semi-directifs ont été conduits auprès de responsables d'agences et d'ETT situées sur les territoires du Nord de la Lorraine et du Sud du Luxembourg : huit responsables d'agences (quatre agences Adecco, deux agences Manpower, et deux agences Vedior-Bis) et quatre responsables d'entreprises d'intérim (Sofitex, Interface, Randstad-Luxembourg, Manpower-Luxembourg). Ces entretiens avaient pour but de cerner le rôle des agences d'intérim sur le marché du travail du bassin transfrontalier de Longwy. Parmi les huit thèmes, deux concernaient les pratiques de formation et leur rapport à la gestion de la main-d'œuvre : les politiques de formation des intérimaires, les politiques salariales, de primes et les actions de valorisation (1) (BELKACEM, 2001 et 2002).

- En 2007, dix responsables d'entreprises et agences d'intérim situées au sein des zones frontalières de la Lorraine ont également été enquêtés (entreprises et agences suivantes : Interface, Turbolux, Kelly-Luxembourg, Rowlands RTT, DLSI, Manpower-Luxembourg, Adecco-cadre, Adecco-BTP, Adecco-Tertiaire, Adecco-Industrie). L'objet de la recherche portait cette fois-ci sur le rôle des ETT dans la régulation du marché du travail au Luxembourg (2). Là encore, le questionnaire comportait une série de questions sur les pratiques de formation : budget annuel de formation, nombre d'intérimaires formés, profil des formés, domaines et organisation de la formation (3).

(1) Les autres thèmes étaient les suivants : identification de l'opérateur (statut juridique, date de création, événements marquants), zone géographique d'activité, critères déterminant le choix d'implantation et perception de la frontière, modes de prospection, rapports aux EU, politiques de recrutement et de détachement. Recherche financée par l'Institut lorrain des sciences du travail, de l'emploi et de la formation.

(2) Recherche financée par la Commission européenne.

(3) Cette enquête a été complétée par une série d'entretiens approfondis auprès de responsables de l'intérim dans les zones frontalières de Longwy et de Thionville. Au cœur de ces entretiens, quatre sujets : caractéristiques de l'agence ou de l'entreprise, modalités de recrutement et de détachement, gestion des relations intérimaires/agences, rapports aux EU. Voir BELKACEM et al., 2007.

financier collecté(4). L'objet de cet article est d'analyser les formes, les évolutions et les limites de la formation dans cette branche d'activité. Ces limites s'expliquent par la nature même de l'inté-

(4) Le rapport d'activité 2010 du FAF-TT (2011c) indique que les sommes collectées, assises sur la masse salariale, passent de 3,2 millions d'euros en 1985 à 40,7 millions en 1993, 122,7 millions en 2003 et 148,3 millions en 2011 (pour cette dernière année, $89 \%$ des fonds sont affectés à la formation; la quantification pour 2011 figure dans le rapport d'activité 2010). Les ETT de plus de dix salariés ont deux options de contribution financière au FAF : dans l'option 1, elles peuvent verser de 50 à $100 \%$ de leur contribution, dans l'option 2 de 7 à $50 \%$. N'ayant pas toujours les moyens d'organiser elles-mêmes les actions de formation, les deux-tiers des ETT choisissent l'option 1 qui fait appel davantage au soutien du FAF-TT. rim puisqu'il s'agit d'une activité économique qui opère le plus souvent dans l'urgence : fournir dans des délais brefs des travailleurs et des qualifications aux clients, les entreprises utilisatrices (EU). Pour traiter ces différentes questions, nous mobiliserons les résultats issus d'une exploitation des données émanant du FAF-TT. Nous mettrons également à contribution les résultats d'enquêtes (voir encadré 1). Afin de définir à la fois le cadre et l'objet de notre analyse, nous commencerons par discuter les principaux résultats de travaux sur le sujet. Nous nous interrogerons ensuite sur le processus de construction du cadre institutionnel de la formation des intérimaires en France, ce qui nous permettra de faire un état de l'importance de la formation, de 
ses évolutions et de ses formes pour, enfin, nous interroger sur ses apports et sur ses limites pour les travailleurs et aussi pour les ETT.

\section{La formation : un objet de recherche récent et un cadre institutionnel évolutif}

\section{La formation des intérimaires : un objet de recherche récent}

Bien que les stratégies de gestion des ressources intérimaires intègrent ou affichent de manière régulière, au moins depuis le début des années 1980, la formation professionnelle continue dans leur contenu, notamment dans les grands groupes du travail temporaire (BeLKacem, Michon, 2011) ${ }^{(5)}$, l'économie et la sociologie du travail, mais aussi les travaux en gestion, ne se sont emparés de la question de la formation des intérimaires que relativement récemment.

\section{Des travaux d'abord centrés sur le rapport intérimlbesoins de production}

Durant les années 1980, le travail intérimaire va surtout être étudié comme un instrument de flexibilité dans le fonctionnement des marchés du travail (Michon, 1986, 1987), en particulier comme un moyen rapide d'adapter le niveau des effectifs salariés aux besoins de production, l'aléa économique et productif étant jugé déterminant. Pourtant, François Michon précise que cette adaptation entre effectifs et besoins de production peut s'opérer de différentes façons. Elle peut porter sur les volumes de travailleurs (flexibilité quantitative) : le recours à l'intérim permet alors d'assurer une adéquation entre effectifs de salariés et niveaux de production. Elle peut concerner également les caractéristiques professionnelles des travailleurs (flexibilité qualitative) : l'utilisation de ces travailleurs permet ainsi un ajustement entre les qualifications des salariés et la nature des besoins de production. Joseph BRESSI (1986), dans cette logique d'adaptation et d'adaptabilité, va jusqu'à considérer les ETT comme des institutions de transformation de la main-d'œuvre. Mais l'usage du travail intérimaire peut aussi devenir constitutif des modes de gestion de la main-d'œuvre. C'est au sein de ces analyses que le questionnement sur la formation dans l'intérim va prendre sens, en particulier sur le rôle qu'elle joue ou peut jouer dans les processus d'adaptation de la main-d'œuvre aux conditions de production.

(5) Notons que l'on peut considérer comme à l'origine du travail intérimaire les écoles de mécanographie qui existaient aux États-Unis au début du siècle dernier : leurs caractéristiques sont en effet voisines de celles des agences d'intérim actuelles. Leur fonction était triple : détachement de personnel, placement de travailleurs auprès d'entreprises clientes et formation des salarié(e)s.

\section{La formation, un élément de l'offre de services proposés par les agences d'intérim aux entreprises utilisatrices}

En France, à notre connaissance, la première recherche sur le sujet a été menée par Dominique Dieppedale, Pierre PaQuier et Hugues Puel en 1993. Elle s'appuie sur une enquête auprès d'une cinquantaine d'intérimaires du secteur du bâtiment et travaux publics et a mobilisé les données du FAF-TT. Les auteurs ont montré que la formation contribuait à faire des agences d'intérim des producteurs d'activités de service élargies pour les EU (Dieppedale et al., 1993, p. 25). À partir d'enquêtes monographiques et d'entretiens approfondis auprès de responsables d'ETT, complétés par des entretiens avec des responsables syndicaux, les travaux de Gilbert LefÈvre, François Michon et Mouna VIPREY ont examiné les orientations stratégiques du secteur de l'intérim en matière d'offre de services et de gestion de la main-d'œuvre. L'un des résultats essentiels est que la formation est utilisée pour fidéliser les intérimaires les plus expérimentés. Étudiant de façon détaillée les pratiques de formation, les travaux de Martine MöBus (2006) vont apporter quant à eux des éléments d'analyse approfondis sur les outils de formation développés au sein de cette branche d'activité, y compris en termes de quantification.

\section{La formation et la gestion en réseau des ressources humaines}

D'autres travaux vont considérer la formation comme l'une des dimensions de l'organisation de la fonction «gestion de ressources humaines intérimaires» (GRHI), participant de la constitution d'un mode de fonctionnement en réseau. Dans cette configuration, certains éléments de la GRH (comme la formation, le «sourcing»(6) ou l'«outplacement» $\left.{ }^{(7)}\right)$ seraient pris en charge par une ou plusieurs ETT associées aux projets de production des entreprises. C'est ce sur quoi insiste par exemple Jocelyne ABRAHAM (1998), pour qui la mise en œuvre de la formation s'inscrit dans le cadre d'un partenariat entre les agences d'intérim et les EU. Rachid Belkacem, Mohamed Bennoui et Régis Rouyer (2007) relativisent fortement cette démarche GRHI, ainsi que l'impact et le contenu du fonctionnement en réseau. Ainsi, en dépit de réels changements, il est notable que l'activité de formation ne concerne en pratique qu'un faible nombre d'intérimaires par rapport aux flux de travailleurs qui transitent par les ETT. Les auteurs font également observer que les autres dimensions de la GRHI sont tout aussi limitées : les promotions sont ainsi inexistantes et les

(6) Recherche de candidats aux profils demandés par les clients. (7) À la demande des EU qui veulent se séparer de certains de leurs employés, les ETT peuvent participer à leur réinsertion professionnelle. 
politiques salariales de faible envergure. BELKACEM (1997) montrait déjà que, dans ces configurations en réseaux d'entreprise, les ETT étaient dans une situation d'autonomie contrôlée vis-à-vis des EU parce qu'elles restaient, dans tous les cas, tributaires des choix de ces dernières et de leurs décisions de former ou de ne pas former des travailleurs. Cette situation d'autonomie contrôlée des ETT par les EU peut d'ailleurs conduire à des processus de quasiintégration des premières au sein des secondes.

\section{La formation, intermédiation et quasi-intégration des agences d'intérim aux $E U$}

Les agences d'intérim peuvent opérer comme de véritables sous-traitants des EU. Elles peuvent même évoluer vers des fonctions quasi-intégrées au sein des EU (BELKACEM, 1997, 2001). Il s'agit là en quelque sorte d'une phase ultime du partenariat dont la traduction est l'implantation, au sein-même des EU, de cellules ou de services dédiés à la gestion des intérimaires (des implants), ainsi que l'illustrent de manière significative les pratiques des très grandes entreprises du secteur de l'automobile. Dans ce contexte, la formation des travailleurs devient une fonction qui peut être prise en charge totalement par les agences d'intérim. Dans un champ d'application distinct, Christine Guégnard, Marie-Claude Rebeuh et Emmanuel Triby (2008) estiment que le développement de la formation par les agences d'intérim renforce leur intermédiation sur un territoire donné(8). En fournissant des ressources, les ETT contribuent à réduire l'incertitude à laquelle font face les EU en leur permettant de gérer les aléas liés à l'activité de production et agissent ainsi sur l'articulation concrète des temporalités. En effet, elles introduisent une continuité temporelle en combinant dans leur offre des séquences de formation et de mission répondant aux variations de l'activité des EU. Elles interviennent donc pleinement dans les dynamiques d'organisation des territoires locaux.

\section{Formation et trajectoires d'emploi}

D'autres travaux de recherche ont étudié les modalités d'accès à la formation des travailleurs précaires, parmi lesquels les intérimaires. Les études de Coralie Perez et Gwenaëlle Thomas (2005) puis de Coralie Perez $(2007,2009)$ ont permis de mieux comprendre leurs déterminants et les pratiques en la matière. S'appuyant sur l'enquête formation continue 2000, réalisée conjointement par l'Insee et le Cereq, elles ont identifié sept trajectoires-types de salariés exposés à la précarité, dont celle dominée

(8) Cette recherche s'est appuyée sur des entretiens semidirectifs auprès de responsables de dix-sept entreprises, quatre agences de travail temporaire (TT) et deux agences locales de l'emploi. Les auteurs ont mobilisé différentes sources d'information disponibles au niveau local dans quatre territoires (Haguenau, Wissembourg, Chalon-sur-Saône et Beaune). par l'intérim ${ }^{(9)}$ (Perez, Thomas, 2005, pp. 112-114). Elles mettent en évidence le fait que la trajectoire d'emploi précaire pénalise l'accès à la formation. Sur la période mars 1998 - mars 2000, 34,5\% des travailleurs inscrits dans des trajectoires dominées par l'intérim ont eu accès à une formation, alors que tel est le cas pour $46,5 \%$ des travailleurs sous contrat à durée indéterminée (CDI) à temps complet (pp. 116-117). Lorsque les intérimaires suivent une formation, il s'agit le plus souvent de programmes d'adaptation aux postes de travail (p. 123). Les formations longues de type CIF (congé individuel de formation) ou bien BC (bilan de compétences), ont tendance à être réservées aux travailleurs que l'ETT cherche à fidéliser, y compris pour leur proposer des missions plus qualifiées. À l'opposé, le non-accès à la formation s'explique surtout par l'absence de propositions émanant du/des employeurs en raison de la précarité du lien salarial (Perez, 2009, p. 11 et 15) : nous ne manquerons pas de revenir sur ce dernier point.

\section{La construction du cadre institutionnel de la formation des intérimaires à partir du début des années 1980}

Depuis le début des années 1980, la construction du cadre institutionnel de la formation dans le secteur de l'intérim en France s'est faite de manière progressive et a procédé d'une double démarche : d'une part, des dispositifs déjà existants au sein des politiques publiques de l'emploi furent adaptés à la situation spécifique des intérimaires; d'autre part, des dispositifs propres à la profession furent mis en place.

\section{Un cadre institutionnel négocié entre les partenaires sociaux}

L'investissement du secteur dans la formation s'est accentué au début des années 1980, dans un contexte de difficultés de recrutement de main-d'œuvre qualifiée dans plusieurs activités économiques, particulièrement (mais pas seulement) dans la construction et le bâtiment.

Les pratiques conventionnelles furent, formellement, l'une des sources majeures de l'élaboration du cadre institutionnel. Conclu le 9 juin 1983 entre

(9) Pour définir ces trajectoires, les auteurs ont retenu les situations d'emploi dominant entre mars 1997 et mars 2000. En s'appuyant sur les données Cereq 2000, ils ont distingué sept profils de trajectoires qui se décomposent en cinq trajectoires stables, une autre trajectoire regroupant un halo de situations de précarité parmi lesquelles figurent le contrat à durée déterminée (CDD), l'intérim, et, enfin, le contrat à durée indéterminée (CDI) à temps partiel. Les cinq trajectoires stables sont dominées par les indépendants, les fonctionnaires, les femmes au foyer, les retraités et les CDI. 
les organisations patronales Promatt et Unett $(10)$ et des organisations syndicales de salariés (CFDT, CFTC, CGC et CGT-FO) ${ }^{(11)}$, le premier accord en établit les fondements avec la création du FAF-TT. Inchangées jusqu'à aujourd'hui, les fonctions de ce fonds sont les suivantes : collecter les contributions obligatoires des ETT à la formation professionnelle continue; s'assurer que le contenu de la formation dispensée au salarié corresponde «bien à des débouchés sur le marché du travail» (art. 11. 15 de cet accord); donner un avis sur la procédure de formation et rembourser à l'ETT les coûts de la formation suivie par le salarié. Cet accord a également, dans le cadre de la loi, précisé les conditions d'accès au congé individuel de formation (CIF).

L'une des questions à laquelle ont été confrontés les partenaires sociaux fut la suivante : quel statut aura l'intérimaire durant la période de formation? La réponse ne releva pas du registre conventionnel mais fut apportée par une loi du 24 février 1984 stipulant que les périodes de formation sont assimilées à des missions. Les contrats de mission-formation constituent ainsi le support de la mise en œuvre des dispositifs de formation. Cependant, pour y avoir droit, des conditions d'ancienneté furent définies : il fallait ainsi justifier d'une ancienneté d'au moins 507 heures d'intérim au cours des douze derniers mois.

Dans la continuité de cette loi, les partenaires sociaux ont conclu d'autres accords importants, dont celui du 16 juin 1986, étendu le 13 janvier 1987, qui concerne l'insertion professionnelle des jeunes intérimaires et adapte à l'intérim les deux contrats en alternance prévus par l'accord national interprofessionnel du 26 octobre 1983 et repris par la loi du 24 février 1984 : les contrats de qualification (mobilisés surtout pour la formation des intérimaires) et les contrats d'adaptation (utilisés principalement pour la formation des salariés permanents).

Les négociations entre partenaires sociaux se poursuivirent. Entériné par la loi du 16 juillet 1990, l'accord du 24 mars 1990 fixa à $2 \%$ de la masse salariale la contribution des ETT à l'effort de formation (l'obligation légale étant alors de $1,2 \%)$. Concernant les CIF, cette contribution fut fixée à $0,3 \%$ (LiAISONS SOCIALES, 1990). En contrepartie de cette hausse, l'État apporta son concours au financement des CIF des intérimaires les moins formés. Un nouvel accord de branche du 15 octobre

(10) L'Unett (Union nationale des ETT, créée en 1976) et le Promatt (Syndicat des professionnels du travail temporaire, créé en 1977) furent pendant longtemps les deux principales organisations patronales dans l'intérim. En 1998, elles ont fusionné pour former le SETT (Syndicat des entreprises de travail temporaire) devenu, en juin 2006, le Prisme (Professionnels de l'intérim, services et métiers de l'emploi). (11) Confédération française démocratique du travail; Confédération française des travailleurs chrétiens; Confédération générale des cadres; Confédération générale du travail - Force ouvrière.
1991 élargit les objectifs de l'accord de 1983 et attribua à la formation le rôle d'enrichissement des capacités «d'employabilité» et de développement des compétences professionnelles des intérimaires. Cet accord se substitue à ceux de 1983 et 1986 (à l'exception des règles de 1983 relatives à la création du FAF-TT). Il précise les instruments existants, notamment le contrat de mission-formation (art. L. 124-21 du Code du travail) et le CIF. Il catégorise les travailleurs intérimaires au regard des dispositifs existants afin de les adapter. En pratique, les CIF sont destinés aux salariés ayant une ancienneté importante dans l'intérim et aux victimes d'un accident du travail. La mission-formation peut être utilisée pour la réinsertion de salariés lorsqu'ils sont nombreux à arriver en fin de détachements dans un bassin d'emploi donné (Liaisons Sociales, 1991). Cet accord affiche à la fois des objectifs économiques et sociaux : adaptation de la qualification aux besoins du marché du travail, organisation d'un déroulement de carrière, reconversion des salariés temporaires victimes d'un accident du travail ou d'une maladie professionnelle, adaptation à l'évolution des normes de sécurité, réinsertion des salariés temporaires en fin de mission dans un même bassin d'emploi, insertion des travailleurs handicapés. Ont été également intégrés le congé de bilan de compétences $(\mathrm{CBC})$ et le contrat d'orientation qui remplace

\section{Encadré 2}

\section{Dispositifs de formation spécifiques à l'intérim}

À la fin de 2004, le contrat de développement professionnel intérimaire $(\mathrm{CDPI})$ a remplacé le contrat de mission jeune-intérimaire (CMJI). Le CDPI concerne toute personne travaillant dans l'intérim au moins 450 heures dans les douze derniers mois et détenant un niveau de qualification inférieur ou égal à un diplôme de niveau V. II s'agit toujours d'une formation qualifiante d'une durée comprise entre 175 heures et 450 heures. C'est un contrat de mission-formation (alternant des périodes de formation et de mission) qui s'adresse aux intérimaires ayant ainsi de l'ancienneté dans l'intérim. À l'issue de sa formation, l'intérimaire peut accéder à un premier niveau de qualification ou bien à une qualification différente, voire complémentaire.

Toujours fin 2004, le contrat d'insertion professionnelle intérimaire (CIPI) a remplacé le contrat de mission formation intérimaire (CMFI). Le CIPI concerne les jeunes sans expérience et sans conditions d'ancienneté, mais aussi des publics rencontrant des difficultés d'insertion ou de réinsertion en raison de l'âge, d'un handicap, d'une situation familiale, sociale ou professionnelle désavantageuse. D'un volume horaire de 210 à 420 heures, en continu, ce dispositif propose également une alternance entre des périodes de formation (théorique ou pratique) et des périodes de mission. 
Tableau 1 : Les principales caractéristiques des dispositifs institutionnels de formation en vigueur dans l'intérim

\begin{tabular}{|c|c|c|c|}
\hline Dispositifs & Public visé & Conditions d'accès & Objectifs \\
\hline $\begin{array}{l}\text { CDPI - Contrat de développe- } \\
\text { ment professionnel intérimaire (en } \\
\text { remplacement du CMJI) }\end{array}$ & Intérimaire quel que soit l'âge & $\begin{array}{l}210 \text { h. dans l'intérim dans les } 12 \\
\text { derniers mois }\end{array}$ & $\begin{array}{l}\text { Améliorer le niveau de qualification } \\
\text { Accéder à des missions plus qualifiées }\end{array}$ \\
\hline $\begin{array}{l}\text { CIPI - Contrat d'insertion profes- } \\
\text { sionnelle intérimaire (en remplace- } \\
\text { ment du CMFI) }\end{array}$ & $\begin{array}{l}\text { Éloigné de l'emploi } \\
\text { Moins de } 26 \text { ans sans expérience } \\
\text { ou } \\
\text { Moins de } 210 \mathrm{~h} \text {. travaillées dans } \\
\text { les } 6 \text { derniers mois }\end{array}$ & Pas de conditions d'ancienneté & $\begin{array}{l}\text { Favoriser l'insertion des personnes les } \\
\text { plus éloignées de l'emploi } \\
\text { Accès à un niveau supérieur de } \\
\text { qualification }\end{array}$ \\
\hline $\begin{array}{l}\mathbf{C P} \text { - Contrat de professionnalisation } \\
\text { en remplacement des trois contrats } \\
\text { en alternance antérieurs : qualifica- } \\
\text { tion, adaptation, orientation }\end{array}$ & $\begin{array}{l}\text { Jeunes de moins de } 26 \text { ans } \\
\text { ou } \\
\begin{array}{l}\text { Demandeurs d'emploi de plus } \\
\text { de } 26 \text { ans }\end{array}\end{array}$ & Pas de condition d'ancienneté & $\begin{array}{l}\text { Maintien dans l'emploi } \\
\text { Insertion - Réinsertion } \\
\text { Acquisition d'une qualification } \\
\text { reconnue }\end{array}$ \\
\hline $\begin{array}{l}\text { CIF - Congé individuel de } \\
\text { formation }\end{array}$ & Intérimaires expérimentés & $\begin{array}{l}1600 \text { h. dans l'intérim au cours } \\
\text { des } 18 \text { derniers mois dont } \\
600 \text { h. dans l'ETT qui signe } \\
\text { l'autorisation }\end{array}$ & $\begin{array}{l}4 \text { objectifs : } \\
\text { - Accès à un niveau de formation } \\
\text { supérieur } \\
\text { - Perfectionnement professionnel } \\
\text { - Changement d'activité ou de } \\
\text { profession } \\
\text { - Ouverture à la culture et à la vie } \\
\text { sociale }\end{array}$ \\
\hline $\mathbf{C B C}$ - Congé bilan de compétences & Intérimaires expérimentés & $\begin{array}{l}3200 \mathrm{~h} \text {. dans l'intérim au cours } \\
\text { des } 36 \text { derniers mois dont } \\
1600 \mathrm{~h} \text {. dans l'ETT qui signe } \\
\text { l'autorisation }\end{array}$ & $\begin{array}{l}\text { Analyse des compétences profession- } \\
\text { nelles, personnelles, motivations, en } \\
\text { vue d'un projet professionnel ou de } \\
\text { formation }\end{array}$ \\
\hline $\begin{array}{l}\text { DIF - Droit individuel à la } \\
\text { formation }\end{array}$ & Intérimaires expérimentés & $\begin{array}{l}2700 \text { h. dans l'intérim au cours } \\
\text { des } 24 \text { derniers mois dont } \\
2100 \text { h. dans l'ETT où la } \\
\text { demande est faite }\end{array}$ & $\begin{array}{l}3 \text { objectifs : } \\
\text { - Élever le niveau de qualification (ou } \\
\text { perfectionnement) } \\
\text { - Validation des acquis de l'expérience } \\
\text { professionnelle } \\
\text { - Bilan et orientation professionnelle }\end{array}$ \\
\hline $\begin{array}{l}\text { VAE - Validation des acquis de } \\
\text { l'expérience }\end{array}$ & Intérimaires expérimentés & 3 ans d'expérience & $\begin{array}{l}\text { Valider un diplôme en lien avec les } \\
\text { acquis professionnels }\end{array}$ \\
\hline
\end{tabular}

le stage d'insertion dans la vie professionnelle (SIVP). Une clause de «partenariat-formation» qui lie «l'investissement formation» à des règles garantissant à l'ETT de ne pas «perdre sa mise» fut introduite. Ces règles consistent à contraindre l'intérimaire nouvellement formé à rester à la disposition de l'ETT, pour réaliser des missions pour son compte, afin qu'elle puisse tirer avantage de l'effort financier consenti en matière de formation. Des indemnités versées par l'intérimaire aux ETT furent prévues en cas de démission de ce dernier.

De nouvelles négociations collectives aboutirent le 22 avril 1994 à la mise en place d'un nouveau dispositif de formation à destination du public jeune (moins de vingt-six ans). Il s'agit du contrat de missions jeunes-intérimaires (CMJI, voir encadré 2), dispositif très prisé par les ETT comme nous le verrons en détail un peu plus loin. Le 9 juin 2003, la profession lança un second type de contrat (à côté du CMJI), se voulant spécifique à la profession, le contrat de mission-formation insertion (CMFI) en direction cette fois des publics éloignés de l'emploi. À la suite de l'accord interprofessionnel sur la formation professionnelle de la fin $2003^{(12)}$, ces dispositifs furent amenés à évoluer.

(12) Loi du 4 mai 2004 relative à la formation tout au long de la vie et au dialogue social.

\section{Les adaptations récentes : simplification et transformation des conditions d'accès aux dispositifs de formation}

Suite à la loi du 4 mai 2004 relative à la formation tout au long de la vie et au dialogue social, les partenaires sociaux vont adopter de nouvelles règles à l'intérieur de la branche de l'intérim (accord signé le 8 juillet 2004) qui ont pour but de permettre de simplifier les dispositifs de formation, d'améliorer les conditions d'accès des intérimaires aux dispositifs de formation en fonction de leur profil, de mieux intégrer et servir les besoins des EU en matière de qualification des travailleurs. Les principales évolutions sont présentées dans le tableau 1.

Ces dispositifs de formation sont variés et leurs modes opératoires différents selon le profil des intérimaires et leur ancienneté dans l'intérim. En pratique, les personnes les moins diplômées mais qui ont une expérience dans l'intérim sont plutôt orientées vers le CDPI plutôt que vers le contrat de professionnalisation (FAF-TT, 2011a). Selon la responsable de la commission sociale du syndicat des ETT de l'époque, le CIF est surtout réservé aux intérimaires justifiant d'une plus longue expérience (MÉDIA-SETT, 2004, pp. 8-9) et permet de les fidéliser afin de stabiliser le noyau dur des intérimaires les plus qualifiés dans un contexte de 
forte concurrence entre les agences d'intérim pour se les accaparer. Les domaines professionnels visés par ces formations sont également très variés. Ils sont fonction des besoins des EU mais également des caractéristiques des bassins d'emploi. Nous pouvons ainsi trouver des formations aux métiers de télé-conseiller ou de préparateur de commandes pour le CIPI; au CQPM (Certificat de qualification paritaire de la métallurgie) soudeur ou au certificat de conducteur routier pour le CDPI; tandis que le CP donne accès à des BTS gestion de PME-PMI, d'informatique de gestion ou au CQPM soudeur ou électro-bobinier. En même temps qu'est favorisée l'intégration ou la réinsertion professionnelle des jeunes de moins de vingt-six ans et des demandeurs d'emploi, ces actions de formation contribuent à la construction de qualifications en adéquation avec les besoins des entreprises sur un territoire donné.

\section{Quelles pratiques de formation dans l'intérim?}

La branche de l'intérim use aujourd'hui d'une panoplie de dispositifs de formation (voir tableau 1) dont la construction a relevé d'une double démarche d'adaptation des outils de formation de droit commun à la situation spécifique des intérimaires (CP, CIF, CBC, DIF, VAE) et d'élaboration de dispositifs de formation spécifiques (CDPI, CIPI, etc.; voir encadré 2) pour faire face à des besoins urgents dans certains domaines professionnels (logistique, métiers du bâtiment, centres d'appel, etc.).

\section{Une démarche d'adaptation à l'intérim de dispositifs de formation existants}

Dans les discours des responsables de la branche, la formation apparaît nécessaire et occupe une place de choix. Ainsi, un des anciens présidents du syndicat des ETT, connu aujourd'hui sous la dénomination Prisme, déclarait : «la formation professionnelle a toujours été une priorité pour la profession. [...] Ayant une réelle connaissance des bassins d'emploi, (les ETT sont) capables de proposer des actions de formation à leurs intérimaires en adéquation avec les besoins en compétences des entreprises. (Ces actions) améliorent l'employabilité des intérimaires (et permettent) une intégration sur le marché du travail» (Media-Sett, 2004, p. 3). Les contrats de qualification (CQ), puis le contrat de professionnalisation $(\mathrm{CP})$ ont été les dispositifs de formation les plus utilisés dans l'intérim (voir tableau 2 et graphique 1). Les responsables d'agence d'intérim rencontrés ${ }^{(13)}$ avancent deux raisons principales expliquant leur intérêt pour ces contrats en alternance : leur durée de formation étant relativement courte, ils permettraient de répondre rapidement aux besoins en qualifications des EU; ils offrent par ailleurs l'opportunité de constituer des viviers de qualifications en fonction des caractéristiques du tissu productif local.

Tableau 2 : Effectifs entrés dans les principaux dispositifs institutionnels de formation intérimaire financés par le FAF-TT de 1990 à 2011

\begin{tabular}{|c|c|c|c|c|c|c|c|}
\hline & $\begin{array}{c}\mathrm{CQ} \\
\mathbf{C P} \\
\text { intérim } \\
\left.{ }^{*}\right)\end{array}$ & $\begin{array}{l}\text { CMJI } \\
\text { CIPI }\end{array}$ & $\begin{array}{l}\text { CMFI } \\
\text { CDPI }\end{array}$ & $\begin{array}{l}\text { Période } \\
\text { de } \\
\text { profes- } \\
\text { sionnali- } \\
\text { sation }\end{array}$ & $\begin{array}{c}\text { CIF } \\
\text { intérim }\end{array}$ & $\begin{array}{c}\text { CBC } \\
\text { Intérim }\end{array}$ & $\begin{array}{l}\text { VAE } \\
\text { intérim }\end{array}$ \\
\hline 1990 & 1043 & & & & 466 & & \\
\hline 1995 & 1906 & & & & 950 & 25 & \\
\hline 1997 & 1361 & 651 & & & 851 & 24 & \\
\hline 1998 & 2104 & 1335 & & & 926 & 33 & \\
\hline 1999 & 2842 & 1634 & & & 1242 & 60 & \\
\hline 2000 & 2925 & 1527 & & & 1599 & 77 & \\
\hline 2001 & 3689 & 1825 & & & 1701 & 90 & 2 \\
\hline 2002 & 3639 & 2169 & & & 2116 & 101 & n. d. \\
\hline 2003 & 3417 & 2440 & 2171 & & 1991 & 170 & n. d. \\
\hline 2004 & 4214 & 2605 & 3278 & & 1867 & 151 & 38 \\
\hline 2005 & 3572 & 2599 & 4102 & & 2182 & 123 & 74 \\
\hline 2006 & 3733 & 4232 & 4426 & & 2137 & 133 & 72 \\
\hline 2007 & 4324 & 3604 & 4767 & & 2251 & 163 & n. d. \\
\hline 2008 & 6653 & 3449 & \begin{tabular}{|l|l}
6098 \\
\end{tabular} & & 2933 & 230 & 68 \\
\hline 2009 & 2113 & 2488 & \begin{tabular}{|l|l}
4458 \\
\end{tabular} & & 2939 & 278 & 70 \\
\hline 2010 & 1942 & 2996 & 3724 & 3974 & 1974 & 227 & 78 \\
\hline 2011 & 3061 & 3735 & \begin{tabular}{|l|l} 
\\
4427
\end{tabular} & 4436 & 2002 & 219 & 132 \\
\hline
\end{tabular}

${ }^{*}$ ) Dans cette colonne, figurent en caractères non gras les effectifs d'entrée en CQ, en caractères gras, les effectifs d'entrée en CP. Pour l'année 2004, année de transition, le nombre résulte de l'addition du nombre de CQ (3469) et du nombre de CP (745).

n. d. : non disponible.

Sources : FAF-TT (2011b, 2011c, 2012); PrISME, Rapport économique et social annuel de 2001 à 2011

Sur moyenne-longue période, le volume des contrats de formation en alternance est tendanciellement à la hausse, sans qu'existent pour autant une corrélation stricte avec l'évolution du volume d'équivalents temps plein et une régularité dans la tendance (voir graphique 1). Le recours aux contrats de formation en alternance est encore plus sensible à la dégradation de la conjoncture économique que l'évolution du volume d'emploi en équivalent temps plein (voir tableau 2 et graphique 1). Ainsi, 1942 actions de formation dans le cadre du contrat de professionnalisation (CP) ont été réalisées en 2010

(13) En 2001, nous avons réalisé douze entretiens semidirectifs auprès de responsables d'agence et d'ETT dans le cadre d'une recherche qui visait à cerner le rôle des agences d'intérim sur le marché local de l'emploi au sein du bassin transfrontalier de Longwy (voir encadré 1). 
contre 4324 deux ans plus tôt, soit un rapport de 1 à 2,2. Mais les dernières statistiques publiées indiquent $3061 \mathrm{CP}$ pour l'année 2011, soit une progression de $57 \%$.

Graphique 1 : Évolution du nombre de contrats de formation par alternance (FA) signés dans l'année et du nombre annuel d'intérimaires (en volume emploi temps plein - ETP) - Indice base 100 pour l'année 1990

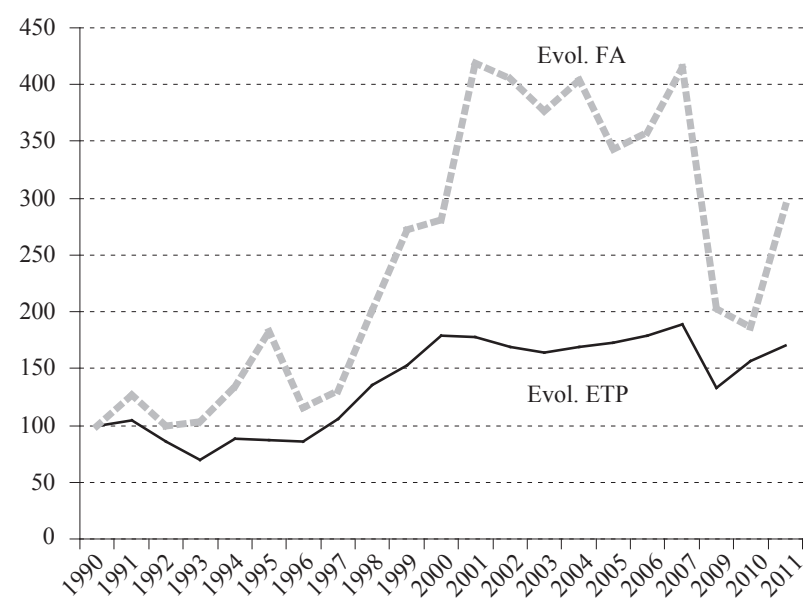

Sources : FAF-Tt (2011b) et PrISme, Rapport économique et social de 1990 à 2011.

\section{Des contrats de professionnalisation ou de qualification pour les salariés peu diplômés, jeunes ou moins jeunes}

La population intérimaire est très majoritairement masculine (71\% des intérimaires), plutôt jeune (âge moyen vingt-neuf ans), ouvrière ( $80 \%$ des intérimaires) et, nous l'avons dit, près de la moitié est peu ou pas qualifiée (45\% des intérimaires) ${ }^{(14)}$. Ces caractéristiques sont relativement stables depuis les années 1980. Si l'on s'appuie sur l'étude menée annuellement par le FAF-TT (voir encadré 1), la répartition hommes-femmes au sein du contrat de professionnalisation est équivalente à celle de l'ensemble des travailleurs intérimaires (voir tableau 3) ${ }^{(15)}$.

(14) Rapport économique et social du secteur de l'intérim (2004 à 2010).

(15) Avec toute la prudence qu'exige la comparaison de caractéristiques sociodémographiques issues de distributions qui ne portent pas sur les mêmes niveaux d'effectifs, nous pouvons observer que l'âge moyen des bénéficiaires intérimaires est plus élevé que celui observé pour ceux de la totalité des contrats au plan national. La classe d'âge 16-25 ans représente $57 \%$ des intérimaires en $\mathrm{CP}$, alors que pour l'ensemble des bénéficiaires tous statuts confondus, elle représente $83,5 \%$.
Tableau 3 : Répartition des CP intérimaire et des CIF intérimaire financés en 2010 par le FAF-TT, par genre, âge, niveau initial de formation à l'entrée et type de validation de la formation (en \%)

\begin{tabular}{|c|c|c|c|}
\hline & $\underset{\text { Intérim }}{\mathbf{C P}}$ & $\begin{array}{c}\text { CIF } \\
\text { Intérim }\end{array}$ & $\begin{array}{c}\text { Ensemble } \\
\text { des intéri- } \\
\text { maires }\end{array}$ \\
\hline \multicolumn{4}{|l|}{ Genre } \\
\hline Hommes & 73 & 82 & 72 \\
\hline Femmes & 27 & 18 & 28 \\
\hline \multicolumn{4}{|l|}{ Âge } \\
\hline 45 ans et plus & 6 & 14 & 14 \\
\hline $26-44$ ans & 37 & 72 & 55 \\
\hline $16-25$ ans & 57 & 14 & 31 \\
\hline \\
\hline I, II et III $(\mathrm{Bac}+2, \mathrm{bac}+3 \mathrm{ou}$ & 14 & 13 & \\
\hline & 27 & 21 & \\
\hline IV (Bac) & 38 & 36 & \\
\hline V (CAP, BEP) & 21 & 30 & \\
\hline \multicolumn{4}{|l|}{$\begin{array}{l}\text { Autre (aucun diplôme, V bis } \\
\text { et VI) }\end{array}$} \\
\hline \multicolumn{4}{|l|}{ Type de validation } \\
\hline \multirow{3}{*}{$\begin{array}{l}\text { Certificat de qualification } \\
\text { professionnelle Qualification } \\
\text { reconnue par une Convention } \\
\text { Collective }\end{array}$} & 11 & 3 & \\
\hline & 61 & 39 & \\
\hline & 28 & 58 & \\
\hline $\begin{array}{l}\text { Titre inscrit au Répertoire } \\
\text { national des certifications } \\
\text { professionnelles (RNCP) }\end{array}$ & & & \\
\hline $\begin{array}{l}\text { Contrats financés par le } \\
\text { FAF-TT en } 2010\end{array}$ & 1942 & 1974 & \\
\hline
\end{tabular}

Source : FAF-ТT, 2011a.

Le contrat de professionnalisation dans l'intérim est principalement orienté vers les publics faiblement qualifiés : $59 \%$ des bénéficiaires intérimaires disposent en effet au mieux d'un niveau initial V (Certificat d'aptitude professionnelle [CAP], Brevet d'études professionnelles [BEP]) à l'entrée du dispositif contre $30 \%$ pour l'ensemble des bénéficiaires du $\mathrm{CP}$ au plan national d'après Ruby SAnchez de la Dares (2011). Les domaines de formation s'inscrivent naturellement dans les secteurs d'activité des EU (métiers du bâtiment et de l'industrie). Avant qu'une action de formation ne débute, et dans la majorité des cas en accord avec l'EU, il est fréquent que plusieurs intérimaires soient envoyés en mission dans les secteurs visés par la formation de façon à évaluer leurs aptitudes et leurs motivations et de sélectionner ainsi les candidats à la formation (LAPOIRE, Puel, 2002).

Selon les responsables d'agences rencontrés ${ }^{(16)}$, les métiers de la logistique et des transports constituent des profils de plus en plus prisés (caristes,

(16) En 2007, nous avons rencontré dix responsables d'entreprises et d'agences d'intérim des zones d'emploi frontalières du Luxembourg (de Longwy et de Thionville) dans le cadre d'une étude financée par la Commission européenne (voir encadré 1). 
chauffeurs de camions, magasiniers, etc.). Le profil intérimaire idéal que recherchent les agences d'intérim est l'intérimaire, plutôt jeune (moins de trente ans) détenant une qualification de niveau CAP/BEP dans les domaines de la mécanique, de la technique et de la soudure notamment : en effet, ces qualifications offrent des possibilités de polyvalence ainsi qu'une capacité importante d'adaptation à des postes de travail différents. Dans le secteur de la construction, on fait par exemple souvent appel à des travailleurs intérimaires pour finir dans l'urgence des chantiers à certaines périodes de l'année. Dans le secteur de l'automobile, beaucoup d'intérimaires travaillent sur les chaînes de production comme opérateurs emboutissage, manipulant des presses pour fabriquer des berceaux de carrosserie et devant s'adapter à des cadences de travail rapides. Dans l'industrie chimique, un secteur traditionnellement utilisateur de main-d'œuvre intérimaire, les missions concernent plutôt des activités de parachèvement, de bardage et de nettoyage industriel. Exposés à des environnements de travail agressifs et intervenant souvent dans l'urgence, les travailleurs intérimaires sont confrontés aux dangers de la manipulation de produits toxiques. Aussi, la formation préalable à une mission est-elle essentielle pour permettre une bonne adaptation du travailleur à son poste ainsi qu'une meilleure connaissance du milieu et des matériaux.

Pour un intérimaire, le contrat de professionnalisation prend la forme d'un contrat à durée déterminée (CDD) d'une durée de six à douze mois (voire d'un maximum de vingt-quatre mois lorsque la formation prépare à un diplôme). Il est articulé autour d'actions de formation assurées par un organisme de formation externe ${ }^{(17)}$, d'une ou plusieurs missions d'intérim et, éventuellement, d'une ou plusieurs périodes de formation. Le montage en est assez complexe et consiste en une personnalisation $\mathrm{du}$ parcours de formation qui alterne moments de formation professionnelle et réalisation de missions d'intérim en adéquation avec la qualification visée. Enfin, ce dispositif couvre l'ensemble des périodes de formation et des missions avec, comme support, un contrat de mission-formation et un contrat de mission. Les jeunes de moins de vingt-et-un ans perçoivent au minimum $55 \%$ du Smic, les personnes âgées de vingt-et-un ans à vingt-six ans $70 \%$ tandis que les plus âgées en perçoivent $100 \%$.

Les formations en alternance sont bien perçues tant par les responsables d'intérim que par leurs clients (les EU), en raison principalement de leur caractère opérationnel. En 2010, la durée moyenne des contrats de professionnalisation s'élève à

(17) En 2011, selon le FAF-TT, les organismes de formation externes mis à contribution pour la professionnalisation des intérimaires sont: AFT IFTIM Formation continue, Promotrans, AFPI Pays de la Loire, Centre de formation professionnelle continue, ASSIFEP Transports, AFPA, etc.
383 heures dans l'intérim selon le FAF-TT (2011a, p. 10) ${ }^{(18)}$. D'après les responsables d'agence rencontrés ${ }^{(19)}$, les contraintes spécifiques de l'activité intérimaire, comme celle d'apporter des solutions aux besoins en qualification des entreprises clientes dans un contexte où la mise à disposition rapide des qualifications demandées est un enjeu de compétitivité pour les ETT, renforcent le caractère opérationnel des actions de formation. À cela s'ajoute le souci permanent d'améliorer la capacité de transferts de qualifications d'une EU à une autre et de constituer un vivier de qualifications dans les domaines demandés.

La dimension opérationnelle de la formation dans l'intérim se traduit également dans le mode de validation de la formation. Seulement $28 \%$ des bénéficiaires intérimaires des contrats de professionnalisation ont obtenu en 2010 un titre inscrit au Répertoire national des certifications professionnelles (RNCP), autrement dit un diplôme, alors que c'est le cas pour plus de $62 \%$ de l'ensemble des bénéficiaires de contrats de professionnalisation au plan national (SANCHEZ, 2011). La majorité des intérimaires $(61 \%)$ obtient ainsi une qualification inscrite dans la classification d'une convention collective (voir tableau 3).

Selon la dernière enquête réalisée en 2010 par l'Observatoire des métiers et de l'emploi $(\mathrm{OME})^{(20)}$, la formation est perçue par les intérimaires comme un important levier de consolidation et d'amélioration de leurs qualifications. Leurs attendus vis-à-vis de la formation (OME, 2011, p. 11) sont l'acquisition d'une qualification supérieure et une élévation corrélée des salaires (29\%), le renforcement des chances de trouver un emploi stable $(28 \%)$ ou tout simplement l'augmentation des opportunités d'emploi (25\%). Pour les jeunes de moins de vingt-six ans, le contrat de professionnalisation est motivé d'abord par la nécessité ou la volonté d'acquérir une qualification supérieure $(35 \%)$ et ensuite par celle d'accroître la probabilité d'accès à l'emploi $(23 \%)$. C'est ce dernier point que privilégient également les personnes de plus de quarante-cinq ans $(40 \%)$ ainsi que la possibilité de changer de métier (34\%). D'autres études confortent ces observations. Selon le FAF-TT (2011a, p. 13), dans les six mois suivant la fin du contrat

(18) Pour l'ensemble des bénéficiaires au plan national qui sont entrés sur ces actions de formation en 2010, cette durée s'élève à 648 heures pour l'année 2010 (DARES, 2011a, p. 5).

(19) Les responsables d'agence et d'ETT rencontrés en 2007 à l'occasion de l'analyse des dimensions transfrontalières du recours à l'intérim (voir encadré 1), ont tous insisté sur les contraintes de l'activité qui tiennent à la nécessité de répondre dans l'urgence à des commandes de main-d'œuvre associées à des compétences.

(20) Cette étude a concerné le suivi d'une cohorte de 5000 intérimaires sur une période de deux ans entre mars 2009 et octobre 2010. Elle avait pour objet l'étude des différents types de trajectoires professionnelles des intérimaires (PrISME, 2011). 
de professionnalisation, $85 \%$ des bénéficiaires ${ }^{(21)}$ sont en activité, parmi lesquels $21 \%$ ont accédé à un contrat à durée indéterminée $(\mathrm{CDI}), 13 \%$ à un contrat à durée déterminée (CDD) et $49 \%$ à un contrat d'intérim. La formation aboutit donc bel et bien, en tendance, à l'élévation du niveau de qualification reconnu. Alors qu'un peu plus de la moitié des bénéficiaires ( $52 \%$ ) étaient ouvriers peu ou pas qualifiés avant la formation, ils ne sont plus que $36 \%$ à l'issue de cette dernière (voir tableau 4).

Tableau 4 : Effets du contrat de professionnalisation sur la structure en termes de catégories socioprofessionnelles (CS) des bénéficiaires (en \%) ${ }^{(*)}$

\begin{tabular}{|l|c|c|}
\hline \multicolumn{1}{|c|}{ Principales CS } & $\begin{array}{c}\text { Avant le } \\
\text { contrat }\end{array}$ & $\begin{array}{c}\text { Après le } \\
\text { contrat }\end{array}$ \\
\hline Agents de maîtrise, cadres & 22 & 23 \\
\hline Employés & 4 & 6 \\
\hline Ouvriers qualifiés & 22 & 35 \\
\hline $\begin{array}{l}\text { Ouvriers non ou peu } \\
\text { qualifiés }\end{array}$ & 52 & 36 \\
\hline
\end{tabular}

(*) Les données de ce tableau proviennent d'une étude commanditée par le FAF-TT (voir encadré 1).

Source : Enquête FAF-TT (FAF-TT, 2011a, p. 17).

Pouvoir travailler dans un emploi stable est la priorité pour la quasi-majorité des intérimaires, alors que leur temps de présence dans l'intérim tend plutôt à s'allonger, faute d'autres meilleures solutions professionnelles. Une étude commandée par la profession (FPE, 2004 : voir MÉdiA-SETT, 2005, pp. 6-9) montrait qu'un cinquième des intérimaires ont ce statut depuis dix ans et un sur trois depuis plus de six ans. L'intérim apparaît ainsi être de moins en moins une situation transitoire. Il constitue aujourd'hui une solution nécessaire pour un peu moins des deux tiers des intérimaires (64\%), parce qu'ils n'ont pas d'autre choix suite aux difficultés rencontrées sur le marché du travail pour trouver un emploi stable (ibid., p. 7). Aussi, la question de la formation des intérimaires est-elle cruciale.

Signe de la forte activité conventionnelle dans ce domaine, le secteur de l'intérim bénéficie depuis janvier 2010, par application d'un accord $\mathrm{du} 10$ juillet 2009, d'un nouveau dispositif de formation : la période de professionnalisation. Ce dispositif permet une action de formation qualifiante combinée à un détachement obligatoire en entreprise, mission qui doit être cohérente avec la qualification visée. Pour accéder à cette formation, les intérimaires doivent justifier d'une ancienneté de 210 heures au cours des douze derniers mois. Le dispositif est en pratique destiné à un public spécifique d'intérimaires, notamment ceux qui alternent fréquemment des périodes de travail et de chômage et ceux dont la qualification est jugée faible au regard de l'évolution des technologies ou des métiers. Il s'adresse également aux travailleurs qui ont vingt ans d'activité professionnelle ou qui sont âgés d'au moins quarante-cinq ans. Selon le FAF-TT, 3974 périodes de professionnalisation ont été financées en 2010 et 4436 en 2011, ce qui correspond à une augmentation de $11,6 \%$, signe de l'appropriation rapide par la profession du dispositif (voir tableau 2). Le rapport économique du FAF-TT pour l'année 2010 précise que le public concerné est celui dont la qualification n'est plus en adéquation avec l'évolution des métiers : $43 \%$ des bénéficiaires intérimaires sont dans cette situation et un quart d'entre eux souhaite accéder à une qualification supérieure. Les actions de formation entreprises sont plutôt de courte durée et, comme précédemment, à visée opérationnelle. Un peu plus des deux tiers de ces actions ne dépassent pas 35 heures de formation (durée moyenne 57 heures). Évolution toute récente encore, avec la loi Cherpion du 28 juillet 2011 pour le développement de l'alternance et la sécurisation des parcours professionnels, les agences d'intérim peuvent également proposer, particulièrement aux jeunes intérimaires, des contrats d'apprentissage d'une durée comprise entre six et trente-six mois.

\section{Les CIF, les CBC et les VAE : des instruments de formation pour les intérimaires les plus expérimentés}

Le secteur s'est également approprié le congé individuel de formation (CIF), le congé de bilan de compétences $(\mathrm{CBC})$ et la validation des acquis de l'expérience (VAE) qui concernent surtout les intérimaires les plus expérimentés. En 2011, plus de 2600 actions de formation relevant de ces trois dispositifs ont été financées (PRISME, rapport 2011, p. 44). Le CIF, action de formation à l'initiative du travailleur est largement majoritaire. Selon les données du FAF-TT (2011b), 2002 intérimaires ont pris un CIF en 2010 contre 466 en 1990 (voir tableau 2). Les CBC n'ont en revanche profité qu'à 219 intérimaires en 2011 au lieu de 25 en 1995. Les CIF sont réservés aux intérimaires qui ont réalisé au moins 1600 heures dans l'intérim au cours des dix-huit derniers mois dont 600 heures dans l'ETT qui signe l'autorisation. Dans un contexte de faible durée des missions (1,7 semaine en moyenne), les conditions d'éligibilité apparaissent très restrictives pour la très grande majorité des intérimaires. Les CIF permettent d'atteindre un ou plusieurs objectifs tels qu'accéder à un niveau supérieur de formation, se perfectionner professionnellement, changer d'activité ou de profession ou s'ouvrir plus largement à la vie sociale et à la culture. D'après

(21) $15 \%$ sont en recherche d'emploi. 
les responsables d'agence ${ }^{(22)}$, le dispositif est relativement bien adapté à l'intérim. Ce sont en général «leurs meilleurs éléments» qui y ont accès. Le CIF permet de les fidéliser alors que le débauchage d'intérimaires expérimentés par la concurrence est une pratique fréquente au sein de marchés du travail locaux très concurrentiels.

Les CIF sont surtout utilisés par des hommes (82\%, contre $72 \%$ dans l'ensemble des intérimaires). Dans la mesure où il est destiné à des publics qui ont plutôt une grande expérience dans l'intérim, il n'est pas étonnant de constater que $86 \%$ des bénéficiaires ont plus de vingt-six ans (voir tableau 3). Les deux tiers ont une formation initiale ne dépassant pas le niveau $\mathrm{V}$, ce qui représente une proportion encore plus forte que chez les bénéficiaires des contrats de professionnalisation (59\%). Les responsables d'agences d'intérim proposent (ou acceptent) des actions de formation, en particulier les plus longues, aux intérimaires qu'ils connaissent bien, ou à ceux susceptibles d'accepter d'autres détachements à la suite de la formation (23). Les CIF sont plus fréquemment sanctionnés que les contrats de professionnalisation par des diplômes inscrits au RNCP : 58 \% des bénéficiaires pour le CIF et $28 \%$ pour le CP (voir tableau 3).

Les formations CIF ont généralement une durée plus longue que celle des autres dispositifs de formation. Le FAF-TT en assure le financement pour une durée maximale de douze mois lorsqu'elle est à temps complet et de 1200 heures sur une période maximale de vingt-quatre mois si elle est à temps partiel. Les formations externes se déroulent au sein d'organismes tels que l'Association pour la formation professionnelle des adultes (AFPA) ou l'AFT IFTIM formation continue dans le secteur

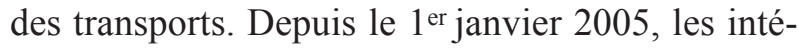
rimaires peuvent également décider de faire jouer leur «Droit individuel à la formation» (DIF), mesure qui existait déjà avant cette date de manière « expérimentale» dans l'intérim. Toutefois, institué par la loi du 4 mai 2004 «relative à la formation professionnelle tout à long de la vie et au dialogue social» (voir tableau 1 ), il semble très peu utilisé encore au sein de l'intérim; il permet aux

(22) Les responsables d'agences et d'entreprises d'intérim que nous avons rencontrés (voir encadré 1), aussi bien à l'occasion de l'étude sur l'impact du travail intérimaire sur le marché local du travail dans le bassin de Longwy (2001) qu'à celle de l'analyse des dimensions transfrontalières du recours à l'intérim à Luxembourg (2007), ont insisté sur la particularité de ces dispositifs qui permettent de stabiliser au sein de l'agence un noyau dur d'intérimaires.

(23) Pratiquement tous les responsables rencontrés en 2001 et en 2007 ont insisté sur ce point. Les expressions utilisées sont très évocatrices. À propos des intérimaires en formation, le responsable d'une agence Adecco située dans la zone d'emploi de Thionville (voir encadré 1) s'exprimait ainsi en 2007 : «ce sont nos cartes de visite auprès de nos clients, (...) notre fonds de commerce (...) nos professionnels ». intérimaires de compléter leur qualification, d'en obtenir une nouvelle ou bien de faire un bilan de compétences ou une VAE.

\section{La création de dispositifs de formation spécifiques à l'intérim}

\section{Des dispositifs institutionnels propres à la branche, qui visent les intérimaires les moins qualifiés}

Comme nous l'avons vu, deux dispositifs de formation propres à la profession furent créés par accords collectifs : le contrat de développement professionnel intérimaire (CDPI) qui a remplacé le contrat de mission-formation jeune intérimaire (CMJI) et le contrat d'insertion professionnelle intérimaire (CIPI) qui a remplacé le contrat de mission-formation insertion (CMFI) (voir encadré 2). Leur création s'explique notamment par la nécessité de pourvoir de manière urgente certaines qualifications.

Le CIPI apporte le support institutionnel indispensable aux nouveaux motifs de recours à l'intérim introduits par la loi Borloo de janvier 2005, qui donne en effet la possibilité aux ETT de conclure des missions d'intérim pour des personnes sans emploi ayant des difficultés sociales et professionnelles particulières qui se voient proposer un complément de formation. Ces dispositifs vont connaître un succès immédiat parce que bien adaptés aux caractéristiques des populations qui passent par l'intérim. Le nombre de bénéficiaires des CDPI va plus que doubler entre 2003 et 2007 en passant de 2171 à 4767 . Il chute à 3724 en 2010 suite à la dégradation de la conjoncture économique pour atteindre 4427 en 2011 (voir tableau 2). Les mêmes variations affectent le CIPI, ce qui souligne la très forte sensibilité de l'intérim à l'évolution de la conjoncture économique. Les décisions de former des intérimaires restent ainsi fortement tributaires des perspectives économiques des EU.

En fonction du profil de l'intérimaire, un arbitrage est souvent opéré entre CP et CDPI. Si l'on compare les bénéficiaires des contrats de professionnalisation à ceux des CDPI, on constate que les CDPI (voir tableau 5) concernent de façon écrasante les hommes (93\% contre $73 \%$ pour les CP) et relativement moins de jeunes de seize à vingt-cinq ans (36\% contre $57 \%$ pour les $\mathrm{CP}$ ). 
Tableau 5 : Répartition des contrats de développement professionnel intérimaire (CDPI) et des contrats de professionnalisation (CP) financés par le FAF-TT en 2010, par genre, âge, niveau initial de formation à l'entrée et type de validation (en \%)

\begin{tabular}{|c|c|c|c|}
\hline Caractéristiques des bénéficiaires & CDPI & CP intérim & $\begin{array}{l}\text { Population } \\
\text { intérimaire }\end{array}$ \\
\hline \multicolumn{4}{|l|}{ Genre } \\
\hline Hommes & 93 & 73 & 72 \\
\hline Femmes & 7 & 27 & 28 \\
\hline \multicolumn{4}{|l|}{ Âge } \\
\hline 45 ans et plus & 7 & 6 & 14 \\
\hline $26-44$ ans & 57 & 37 & 55 \\
\hline $16-25$ ans & 36 & 57 & 31 \\
\hline \multicolumn{4}{|l|}{ Niveau initial de formation à l'entrée du dispositif } \\
\hline IV $(\mathrm{Bac})$ & 14 & 27 & \\
\hline V (CAP, BEP) & 47 & 38 & \\
\hline Autre (aucun diplôme, V bis et VI) & 39 & 21 & \\
\hline \multicolumn{4}{|l|}{ Type de validation } \\
\hline $\begin{array}{l}\text { Certif. de qualif. professionnelle (ou qualification reconnue par une Commission } \\
\text { paritaire nationale pour l'emploi [CPNE]) }\end{array}$ & $\begin{array}{c}8 \\
71\end{array}$ & $\begin{array}{l}11 \\
61\end{array}$ & \\
\hline \multicolumn{4}{|l|}{ Qualification reconnue par une convention collective } \\
\hline Titre inscrit au Répertoire national des certifications professionnelles (RNCP) & 21 & 28 & \\
\hline Durée moyenne de la formation - heures & $286 \mathrm{~h}$ & $383 \mathrm{~h}$ & \\
\hline Contrats financés par le FAF-TT en 2010 & 3724 & 1942 & \\
\hline
\end{tabular}

Source : FAF-TT (2011a).

L'arbitrage entre les deux dispositifs s'opère surtout sur le critère du niveau de qualification. Les personnes les moins diplômées sont en général orientées vers le CDPI auquel les partenaires sociaux de la branche ont attribué le rôle de répondre aux besoins de ces publics peu ou pas qualifiés afin de pouvoir les placer sur les missions proposées. En 2010, $86 \%$ des bénéficiaires détiennent au mieux un diplôme de niveau V (59 \% pour les CP) tandis que 39 \% n'ont aucun diplôme (21 \% pour les $\mathrm{CP}$ ). Autre différence, la durée moyenne de formation dans le cadre d'un CDPI s'établit à 286 heures en 2010 contre 383 heures pour le CP (voir tableau 5). Il s'agit alors principalement de formations dont le but est d'adapter rapidement les intérimaires aux missions proposées; elles sont sanctionnées par une qualification reconnue dans la classification d'une convention collective (71\% pour les CDPI et $61 \%$ pour $\mathrm{CP}$ ) plutôt que par un diplôme (21\% et $28 \%)$.

\section{Également des actions de formation d'appoint de très courte durée}

Les grands groupes de travail temporaire, tels que Manpower, ont également mis en place des outils de formation pour des actions encore plus ciblées ${ }^{(24)}$ comme le perfectionnement des intérimaires avant une mission ou entre deux missions, ou pour les

(24) Les observations qui suivent sont tirées de quelques monographies d'entreprise de TT (voir encadré 1). sensibiliser à la sécurité( ${ }^{(25)}$. Ces outils peuvent adopter des formes variées comme celle du chéquier formation inter-missions que l'on trouve par exemple en Île-de-France. L'intérimaire dispose de vingt-quatre à quatre-vingts heures de formation lors des journées d'inter-missions, par tranche de deux, quatre ou huit heures en fonction de la nature de la formation. L'intérimaire a également la possibilité de se perfectionner à domicile en utilisant 1'outil informatique grâce à des systèmes d'autoformation comme «Téléskill formation» dans le domaine de l'assistance-export et du télémarketing ou grâce encore au catalogue Techtrack TM(26) comportant près de 1400 cours de bureautique et d'informatique. Ces formations peuvent aussi se dérouler dans des centres de formation spécialisés au sein même du groupe de travail temporaire ou encore dans des centres de formation agréés (Afpa, AFT-IFTIM, Greta(27), etc.). Manpower a ainsi créé ses propres centres de formation tertiaire à travers la France; ils dispensent principalement

(25) Par exemple aux normes de sécurité UTE (Union technique de l'électricité) pour le personnel exposé aux risques électriques.

(26) Ce catalogue est disponible via internet sur le Global Learning Center $(G L C)$. Ce dernier donne la possibilité d'accéder à une première offre de formation comprenant des tests pour situer le niveau de connaissances, donne ensuite la possibilité de suivre des cours «on-line» ou en les téléchargeant et enfin permet un accompagnement dans le cadre d'une «hotline» technique, du tutorat ou tout simplement de forums de discussion.

(27) Un Greta est un groupement d'établissements publics d'enseignement qui mutualisent leurs compétences et leurs moyens pour proposer des formations continues pour adultes. 
des formations de bureautique (Word, Excel, Powerpoint) et d'informatique, à la fois pour les utilisateurs et les programmateurs (progiciel SAP, Oracle, BAAN, etc.). Dans le même sens, le groupe Plus intérim a mis en place un centre «Plus micro force» qui assure des entraînements d'une demijournée ou d'une journée pour combler des lacunes identifiées lors des tests de sélection. Ce centre spécialisé dans la formation informatique assure pour les intérimaires aussi bien des formations aux logiciels destinés aux utilisateurs (TTX, tableurs, pré-PAO, PAO, etc.) que des formations systèmes, réseaux, langages, etc. pour le personnel informatique.

\section{Portée et limites de la formation dans l'intérim}

Si le secteur de l'intérim s'est approprié des dispositifs de formation existants et a développé des dispositifs spécifiques, il n'en demeure pas moins, qu'en définitive, peu d'intérimaires accèdent à la formation professionnelle continue, encore moins à des formations qualifiantes de longue durée, au regard des flux de personnes qui passent par les ETT. En 2011, 15659 intérimaires sur une population totale de 576080 en équivalent emplois-temps-plein ont bénéficié d'une action de formation financée par le FAF-TT en vue d'une professionnalisation (comprenant les CP, CIPI, CDPI, périodes de professionnalisation), ce qui représente un taux de $2,7 \%$ (voir le rapport économique et social du PrISME, 2011). Comment peut-on expliquer ces limites?

\section{L'hétérogénéité de la population intérimaire}

Selon les responsables d'agences rencontrés dans nos deux enquêtes, ces formations, même si elles sont de courte durée, représentent un important investissement. Lorsqu'elles ont lieu, elles doivent rapporterun retour sur investissement. Or, la volatilité de la population des travailleurs intérimaires dont la plupart considèrent cette activité comme un moment de passage, une transition uniquement, n'autorise pas toujours un tel investissement. À côté des intérimaires permanents qui constituent entre $15 \%$ et $20 \%$ de l'effectif total selon les agences enquêtées, ceux que les responsables nomment les «intérimaires professionnels», et pour qui le passage par l'intérim est donc un véritable choix, il y a beaucoup d'intérimaires occasionnels qui «au bout de quelques missions disparaissent» ou bien ceux qui ont été formés et "vont monnayer leurs compétences ailleurs $\gg\left({ }^{(28)}\right.$. Les travaux de Colette JoURDAIN (2002, p. 10) illustrent bien l'hétérogénéité de la population des intérimaires. L'auteur distingue

(28) Responsable d'une agence Adecco située dans la zone d'emploi de Longwy (voir encadré 1). cinq schémas de passage par l'intérim : la recherche d'un revenu d'appoint; l'attente de mieux; un choix volontaire; la quête d'un tremplin professionnel et les intérimaires à durée indéterminée. Les travaux de Dominique Glaymann (2008, pp. 37-39) insistent également sur l'hétérogénéité et montrent que le choix du passage par l'intérim reste un choix sous contrainte. Catherine FAURE GUICHARD (1999) va également dans ce sens en distinguant différents motifs de passage par l'intérim : l'intérim d'insertion rend compte par exemple d'un phénomène structurel correspondant à l'entrée sur le marché du travail; il s'inscrit ainsi en début de cycle professionnel à la sortie de l'école pour les jeunes (pp. 4-7). Dans ce type d'intérim, la formation peut trouver tout son sens comme nous l'avons vu à travers les programmes de formation en alternance ou encore les CIPI. L'auteur repère ensuite l'intérim de transition. Elle définit enfin l'intérim de profession, résultat d'un choix délibéré de passage par les services des agences de travail temporaire (pp. 11-15). C'est dans cette dernière catégorie que se trouvent les intérimaires susceptibles de pouvoir suivre des formations relativement longues. Ce sont surtout eux qui font l'objet d'actions de fidélisation de la part des ETT.

\section{L'intérim : une activité fonctionnant dans l'urgence}

Les formations développées dans l'intérim ont une visée essentiellement opérationnelle. Il s'agit avant tout d'adapter rapidement les intérimaires aux postes de travail et de faciliter les transferts de qualifications d'une entreprise à une autre dans un contexte où les durées de missions se réduisent et où les modèles d'organisation du travail en flux tendus se développent. De plus, la succession rapide des missions impliquant des changements fréquents de milieu professionnel, de rythmes de travail, voire de secteurs d'activité n'autorisent pas toujours les approfondissements, les perfectionnements et en définitive les apprentissages d'un métier. Une autre contrainte de la formation dans l'intérim tient à la situation même de la plupart des agences, des petites officines, organisées autour de trois à quatre personnes au maximum, ce qui ne facilite pas le développement d'actions de formation. Une des caractéristiques fondamentales de l'intérim est de répondre dans l'urgence à des demandes en maind'œuvre et/ou en qualification émanant des EU. Le schéma organisationnel des ETT est classiquement structuré autour de cinq principales fonctions qui sont le démarchage auprès des entreprises locales, la définition des besoins en matière de profils de la main-d'œuvre recherchée, l'enregistrement de personnes candidates susceptibles d'être détachées, leur sélection et les détachements effectifs. Aussi, la formation ne devient-elle impérative que lorsque l'agence d'intérim ne dispose pas des profils demandés par les EU, quelles qu'en soient les 
raisons, et apparaît alors comme une prestation de service parmi d'autres.

\section{Un paradoxe de la formation dans I'intérim : qualification de la mission ou qualification de la personne?}

D'après les responsables rencontrés dans nos deux enquêtes, la formation doit répondre à trois impératifs : apporter une réponse aux attentes personnelles des travailleurs qui, en raison de leur situation précaire vis-à-vis de l'emploi, ont, pour deux tiers d'entre eux, la volonté d'accéder à un emploi stable; apporter une réponse aux besoins de production des entreprises soumises aux aléas du marché et qui se doivent donc d'être à la fois compétitives et réactives; enfin, apporter une réponse aux évolutions des besoins de qualification sur un bassin/territoire donné.

En raison du caractère triangulaire de la relation d'emploi dans l'intérim, la question de la formation est enserrée dans des processus paradoxaux. En effet, si l'objet de la formation est d'accroître le niveau de qualification afin d'augmenter, comme le souligne Pierre Naville (1956, pp. 13-14) «la capacité d'exécuter un processus de travail déterminé [...], augmenter l'habileté [dans l'exécution de tâches]» ou, comme le précise Alain d'IrIBARNe (1978, p. 89), afin d'accroître «la capacité individuelle de maîtriser une situation de travail déterminée en faisant appel à son potentiel», la qualification doit être définie en fonction de trois principaux éléments aux déterminants distincts (CHARLIER et al., 2003, pp. 27-28). Le premier élément concerne ainsi la nature du travail à effectuer. La qualification correspond au contenu du travail et aux conditions dans lesquelles il s'exerce (plus ou moins complexe, répétitif, pénible, etc.). Le second élément se rapporte quant à lui à la qualification du type d'emploi caractérisée par le niveau de salaire. À chaque niveau de qualification est associé un salaire accompagné éventuellement de primes et de formes spécifiques de rémunération (individuelles ou collectives, plus ou moins encadrées par des conventions collectives). Enfin, le troisième élément envisage la qualification des personnes du point de vue de leurs qualités, de leurs diplômes, de leurs connaissances, de leurs savoirs, de leur habileté et des compétences qu'elles ont acquises. Or, certains auteurs estiment que le déclassement et la déqualification au regard de la qualification réelle (qui ne se limite donc pas à la qualification scolairement reconnue mais prend en compte les qualifications acquises pendant le parcours professionnel) ne sont pas rares au sein du secteur (GLAYMANN, 2007), dans lequel a pu être mis en évidence un écart important entre qualification $\mathrm{du}$ travail et qualification de la personne. On est alors en droit de s'interroger sur le rôle et le statut à attribuer à la formation dans l'intérim? S'agit-il d'un simple moment afin d'élever la qualification du travail? de l'emploi? ou bien de la personne?

\section{Bibliographie}

Aвraham J. (1998), «Travail Temporaire : éclatement ou partage d'une GRH en réseau», ge Congrès de l'Association de Gestion de Ressources Humaines, IAE de Versailles - St Quentin, 26-28 septembre, pp. 18-34.

BelKacem R. (1997), La relation salariale dans l'intérim, Doctorat en science économique, François Michon (dir.), Université de Paris I - Panthéon-Sorbonne.

BelKACEM R. (2001), «Le recours aux travailleurs intérimaires dans le bassin d'emploi de Longwy : une pratique structurelle », in Del Sol M., Eydoux A., Gouzien A., Merle P., Turquet P. (dir.), Nouvelles dimensions de la précarité, Rennes, Presses universitaires de Rennes, pp. 157-170.

Belkacem R. (2002), «La formation dans l'intérim», Formation \& territoire, ${ }^{\circ} 8$, pp. 24-44.

Belkacem R., Bennoui M., Rouyer R. (2007), Le rôle $d u$ travail intérimaire dans la régulation du marché $d u$ travail au Grand-Duché de Luxembourg, Rapport final, Activités prévues dans le programme Eures 2006-2007/ Convention de subvention Eures VS/2006/0051.
Belkacem R., Michon F. (2011), «L'intérim en France. Où en est-on?», in Belkacem R., Kornig C., Michon F. (dir.), Visages de l'intérim en France et dans le monde, Paris, l'Harmattan, pp. 39-75.

Bressi J. (1986), Intérim et structuration de la maind'œuvre: le cas des entreprises de travail temporaire de Grenoble et d'Annecy, Ronéo, Grenoble.

Charlier M., Colin T., Grasser B., Higelé J.-P., Khristova A., Rose J., Rouyer R., Ryk G. (2003), La construction sociale des frontières entre la qualification et la non qualification, Rapport de recherche, Paris, Dares.

Dieppedalle D., Paquier P., Puel H. (1993), L'intérim dans le BTP, une filière de professionnalisation, Rapport pour le Ministère de l'Équipement, du logement et des transports, Plan Construction et Architectures, Programme Emploi-Qualification-Formation.

FАF-Тт (2002), «Les effets des dispositifs de formation $\mathrm{du}$ travail temporaire sur l'insertion professionnelle des intérimaires », Études, Paris, Fonds d'assurance formation du travail temporaire, avril. 
FAF-TT (2003), Regards croisés sur la formation des intérimaires en Europe. Pratiques des entreprises de travail temporaire, Paris, Fonds d'assurance formation du travail temporaire, rapport final, janvier.

FAF-Tт (2008), La formation des jeunes intérimaires dans le travail temporaire en 2008. Données d'analyse de la professionnalisation et du congé individuel de formation des intérimaires, Paris, Fonds d'assurance formation du travail temporaire.

FAF-TT (2010), «Intérim et formation en France en 2009 », Études, Paris, Fonds d'assurance formation du travail temporaire, mai.

FAF-TT (2011a), «Les effets de la formation sur l'insertion professionnelle des intérimaires », Études, Paris, Fonds d'assurance formation du travail temporaire, juin.

FAF-TT (2011b), «Intérim et formation en France en $2010 »$, Etudes, Paris, Fonds d'assurance formation du travail temporaire, mai.

FAF-Tт (2011c), Rapport d'activité 2010, Paris, Fonds d'assurance formation du travail temporaire.

FAF-TT (2012), «Intérim et formation en France en $2011 », E$ tudes, Paris, Fonds d'assurance formation du travail temporaire, juin.

FAure-Guichard C. (1999), «Les salariés intérimaires, trajectoires et identités», Travail et emploi, $\mathrm{n}^{\circ} 78$, pp. 1-20.

FinOT J. (2011), «L'intérim en 2010 : reprise du travail temporaire», Dares Analyses, $\mathrm{n}^{\circ} 52$.

FinOt J. (2012), «L'intérim en 2011 : croissance soutenue», Dares Analyses, n 42.

Glaymann D. (2007), L’intérim, Paris, La Découverte, Coll. «Repères : Sociologie».

Glaymann D. (2008), «Pourquoi et pour quoi devient-on intérimaire?», Travail et emploi, $\mathrm{n}^{\circ} 114, \mathrm{pp}$. 33-43.

Guegnard C., Rebeuh M.-C., Triby E. (2008), «Entreprises de travail temporaire : former pour renforcer l'intermédiation sur un territoire», Formation emploi, $\mathrm{n}^{\circ} 102$, pp. 41-53.

IRIBARNE A. (d') (1978) «Note complémentaire sur les qualifications et leurs évolutions» in Commissariat général du plan, La qualification du travail : de quoi parle-t-on? Paris, la Documentation Française, pp. 88-96.

Jourdain C. (2002), «Intérimaires, les mondes de l'intérim», Travail et emploi, $\mathrm{n}^{\circ}$ 89, pp. 9-28.

Lefevre G., Michon F., Viprey M. (2002), «Les stratégies des entreprises de travail temporaire, acteurs incontournables du marché du travail, partenaires experts en ressources humaines», Travail et emploi, $\mathrm{n}^{\circ} 89$, pp. 45-64.

LAPOIRE M., Puel H. (2002), L'intérim dans le secteur du $B T P$, une filière de qualification sous valorisée? Étude $d u$ rôle des entreprises de travail temporaire dans le recrutement et la qualification des travailleurs du BTP en Rhône-Alpes, Lyon, Économie \& Humanisme.

Liaisons Sociales (1990), «Contrat à durée déterminée, travail temporaire : accord du 24 mars $1990 »$, Liaisons Sociales, suppl. au $\mathrm{n}^{\circ} 10684$, Législation Sociale, $\mathrm{n}^{\circ} 6360$ du 9 avril 1990, 15 p.

Liaisons Sociales (1991), «Travail temporaire - accord sur la formation», Liaisons Sociales, suppl. au n 6592 du jeudi 14 novembre 1991, 14 p..

Möвus M. (2006), «Le développement des compétences dans le travail temporaire en France : approches et dispositifs », Nef, $\mathrm{n}^{\circ} 23$.

Media-Sett (2004), «Accord de branche du travail temporaire - Les nouvelles perspectives de la formation professionnelle», Média SETT, n 24, pp. 6-9.

Media-Sett (2005), «Étude-FPE-TT. Regard des intérimaires sur l'intérim », Média SETT, n 25, pp. 6-9.

Michon F. (1987), «Flexibilité et marché du travail», Cahiers français, $\mathrm{n}^{\circ} 231$, pp. 35-39.

Naville P. (1956), Essai sur la qualification du travail, Paris, M. Rivière, coll. "Recherches de sociologie du travail».

OME (2011), Regards croisés sur l'intérim, Paris, Observatoire des métiers et de l'emploi, juillet.

Отт (2008), Regards des intérimaires sur l'intérim, Paris, Observatoire du travail temporaire, mars.

Perez C., Thomas G. (2005), «Trajectoires d'emploi précaire et formation continue», Économie et statistique, $n^{\circ} 388-389$, pp. 107-127.

Perez C. (2007), «Emplois précaires et formation continue», Savoirs, 2007/4, Hors-série, pp. 29-46.

Perez C. (2009), «Pourquoi les travailleurs précaires ne participent-ils pas à la formation professionnelle continue?», Formation emploi, n ${ }^{\circ}$ 105, pp. 5-19.

Prisme - SETT, Rapports économique et social 1999 à 2011, Paris, Professionnels de l'intérim, services et métiers de l'emploi.

Prisme (2011), «Trajectoires et devenir de 5000 intérimaires. Les trajectoires professionnelles des intérimaires à l'étude», PRISME le magazine, $\mathrm{n}^{\circ} 20$, pp. 6-9.

SAnchez R. (2011), «Le contrat de professionnalisation en 2010 : légère hausse des entrées », Dares Analyses, $\mathrm{n}^{\circ} 28$. 